\title{
Open Science, Data Analysis, and Data Sharing
}

\section{Daniel R. Isbell, University of Hawai‘i at Mānoa, disbell@ @awaii.edu,} https://orcid.org/0000-0001-5850-8809

\begin{abstract}
Collecting and analyzing data can be arduous, time-consuming labor. Our first instincts might not be to give the data away and reveal the steps behind the 'magic' of analyses. Nonetheless, sharing data and analysis steps increases the credibility and utility of our work, and ultimately contributes to a more efficient, cumulative science. Of course, recognizing the value of data and analysis sharing is one thing - actually doing the sharing is another. Sharing data and analyses is fraught with uncertainties (e.g., What should I share? What can I share? Will my data spreadsheet and analysis script even make sense to someone else?) and, at the end of the day, amounts to additional tasks to be completed. This chapter goes beyond persuading readers to share and presents answers to common questions, advice for best practices, and practical steps for sharing that can be integrated into your research workflow. Easy-to-use, free resources like R, RStudio, and the Open Science Framework are introduced for implementing recommended practices.
\end{abstract}

\section{Introduction}

Sharing data and analysis details are two Open Science practices that enhance the transparency and credibility of research. While applied linguistics research has not (yet) been deemed to be in the midst of a credibility crisis (cf. psychology, Simmons et al., 2011), there are plenty of reasons for us to be concerned, ranging from limited statistical knowledge (Loewen et al., 2020), poor statistical reporting in published research (Al-Hoorie \& Vitta, 2019), and evidence of questionable research practices, and even fraud (Isbell et al., under review). Simply put, when others can peer under the hood of a published research study, it is easier to understand 
exactly what was done and if it was done well, ultimately making it easier to trust the study's findings. Sharing analysis details is also crucial for replication, as it allows other researchers to closely follow your analytical steps so that any differences in outcomes can be more confidently attributed to differences in the samples, contexts of data collection, or deliberate changes/manipulations in study design (see McManus, this volume).

Data and analysis sharing can also be viewed positively from an ethical perspective (see also Sterling, this volume). By sharing your data and analysis scripts, you are helping others be more effective and ultimately facilitating knowledge generation that, however indirectly, might yield greater benefits to people in the 'real world'. For example, sharing your data and analyses with journal reviewers allows them to provide better evaluation and feedback to you and the journal editors (e.g., American Statistical Association, 1999), which helps the peer review process come closer to its ideal as a quality assurance check on knowledge production. Other researchers can also use your data and scripts to generate new insights through re-analysis or synthesis. Hui (2020) is an exemplary case where a researcher was able to re-analyze data from another team (Elgort et al., 2018) and conceptually extend that work in new data collection (Hui also made his new data and analyses openly available: https://osf.io/2ta7m/). Sharing data can also facilitate integrative data analysis (Hussong et al., 2013), where researchers combine data from several studies (e.g., Isbell \& Son, 2021), which in turn can help overcome the issue of small sample sizes in many applied linguistics subdomains (e.g., instructed SLA, Loewen \& Hui, 2021). In research syntheses, open data and analyses can help other researchers recover unreported statistics, more accurately code methodological features of studies, and conduct useful reanalyses of data (Nicklin \& Plonsky, 2020). Students and fellow researchers alike can also learn from your analyses and practice their skills using your data. And while it might not 
happen very frequently, interested members of the public can also more scrutinize your data and analyses when relevant to making policy decisions or educating people on important languagerelated issues. To resort to a cliché, "sharing is caring", and it's also getting the most bang for the buck (not to mention blood, sweat, and tears) when it comes to overall beneficial impacts of the research enterprise.

Despite the apparent benefits of sharing data and analysis details, it also appears that it is not a universal practice among applied linguists, in some part due to the extra effort involved in sharing and concerns about whether sharing original study data is an okay thing to do (Liu \& De Cat, this volume). With the goal of getting more applied linguists on board, the approach to sharing data and analyses presented in this chapter is oriented to achieving a minimum standard of openness and transparency. By minimum, however, I do not mean low: The standard is that any other researcher should be able to access your data and reproduce your reported findings by following your analyses. I want you to be able to achieve that standard with minimal effort and time. I will not exhort you to produce a custom R package that bundles your data and analyses, demand that you use GitHub throughout your entire project, or mandate the creation of an interactive data and analysis dashboard. Instead, I will be encouraging you to use a user-friendly project management and data repository service, the Open Science Framework, (OSF, WwW.osf.io) to share data files and analysis scripts. Last, while this chapter is primarily oriented to quantitative research, it is not my intention to exclude qualitative researchers. Far from it qualitative researchers are encouraged to share data, codes, and coding schemes underlying their interpretive analysis and make available other research materials (e.g., interview protocol) which allow others to more richly understand and scrutinize findings. To that end, I hope qualitative researchers will consider how advice presented here can be applied to their research practices. 


\section{Preparing Data for Others}

\section{What should you share?}

In short, you should share all the raw data from a study and a cleaned set of data that can be 'plugged in' to your primary analyses. Raw data include spreadsheets you download from your online survey, text files from a corpus, and individual output files from experiment software like E-prime, etc. Clean data is created through various processes of collation, transformation, and reformatting. Clean data facilitate human inspection and statistical analysis, but there should always be a clear path from the clean data back to the raw data.

There are some ethical caveats for data sharing. For one, if you are working with data that are already openly available, you do not need to reshare it. This is often the case in corpus linguistic research, which often involves freely available corpora. Additionally, some data may not be yours to share. For example, testing companies may provide data directly to researchers and prohibit any sharing of the data. Finally, perhaps the most difficult aspect of data sharing to navigate is protecting research participants (Meyer, 2018). In general, you should not share (parts of) data that could be used to identify participants or otherwise put them at risk without express permission. Personally identifiable information (PII), like email addresses, IP addresses, and GPS coordinates commonly collected in online surveys, should generally be removed from any shared data or otherwise anonymized (see next section). Specific types of data bring special challenges, like audio/video recordings. In such cases, if express permission to share recordings is not obtained from participants, it is generally fine to share derivative data that is fairly close to the raw data - transcripts of speech recordings, for example, accompanied by acoustic and temporal measures. 


\section{Anonymization}

As mentioned, PII should be removed from shared data or otherwise anonymized. For survey data, removal is often the easiest strategy. For recordings, transcripts, essays, or other extensive language data, anonymization requires more effort. One helpful technique is the use of pseudonyms (assigning fake names to real people) (Saunders et al., 2015). After carefully reviewing your data and identifying all real names, you can use software like R or just the findand-replace function in word processing software to change each real name to a pseudonym this works for shorter transcripts of spoken performances as well as more extensive transcripts of interviews used in qualitative data. Other PII will need to be handled in different ways. Saunders et al. (2015) advise researchers to exercise care when it comes to places, religious/cultural background, occupation, and family relationships (among others) that often come up in the course of interviews. That is not to say all such information must be removed, replaced with pseudonyms, or otherwise obfuscated, but researchers should strive to limit the re-identifiability of subjects in open, shared data (Meyer, 2018). Despite ethical considerations and effort required to address them, though, the key takeaway here is that you shouldn't not share an entire dataset because the raw data contain a few pieces of PII - invest a little time to anonymize the data and then share it.

\section{Data Format}

You should share your data in universal formats whenever possible. For a great deal of data in applied linguistics, this means various types of text files or spreadsheets. For spreadsheet types of data, you should share the data in comma-separated values (.csv) or tab-separated values (.tsv) files (Broman \& Woo, 2018; Buchanan et al., 2021). For transcripts of language data, plain 
text files (.txt) are generally preferred. Encoding is another aspect of file formats that applied linguists should be conscious of. File encodings like UTF-8, for example, make it easier for others to access text data that uses characters outside of the Latin alphabet. Sharing data in universal formats helps ensure that other researchers, regardless of what operating system they use and what software they have available, can actually utilize the data you worked so hard to collect and share.

Accordingly, you should not share data exclusively in proprietary, non-universal formats. That means .sav files exported from SPSS should not be the only format you make your data available in. It also means you should generally avoid common formats like .doc/.docx for text files and .xls/.xlsx for spreadsheet data - despite these Microsoft Office formats becoming increasingly accessible by non-Microsoft software (e.g., Google's online productivity software can open these files), they can still cause problems for some users and there is just no good reason to use them over more universal formats. One way to help ensure that no one will run into a barrier in accessing your shared data is to share it in multiple file formats. Ahn et al. (2017), for example, shared their data on OSF (https://osf.io/b2478/) in Excel (.xlsx) and plain text (.txt) formats.

\section{Raw Data}

Raw (uncleaned) data are the most useful data from a reproducibility and credibility perspective. The credibility of a study can be greatly enhanced by making raw data available, as it allows other researchers to judge the authenticity of the underlying data. In social sciences, suspected data fraud has been flagged by careful analysis of final results that were too good to be true, but ultimately inspection of the raw data provides some of the strongest evidence of authenticity and ultimately a study's credibility (Simonsohn, 2013). Beyond authenticity, sharing 
raw data allows others to see how the raw data was ultimately cleaned (see next section) before being subjected to primary analyses. Doing so also allows other researchers to explore alternative data cleaning or transformation procedures, or incorporate data into an integrative data analysis.

Although you want to keep the raw data as untouched as possible, it is often a good idea to at least give each raw data file a sensible name. In the case of text files in a small corpus, filenames may be quite descriptive, including information about the source of the text and/or the author (e.g., “essay_prompt1_mandarin_P001.txt”). “001.txt” is less helpful. Similarly, for output files from psycholinguistic software, making sure the participant ID is in the filename can be incredibly helpful for others (and yourself), especially when participants may have multiple files for various tasks.

\section{Clean Data}

As valuable as raw data are, researchers all know that a lot of work stands between raw data and data in a format that facilitates analysis. It goes without saying that you give this data a sensible file name - “data.csv" doesn't cut it. Beyond a sensible file name, you should also think about the shape and organization of your clean data as well as how you name variables within the data file.

\section{Tidy Data}

Data should be both clean and tidy. Tidy, for our purposes, refers to the organization of data. Wickham (2014) described three key characteristics of tidy data:

1. Each variable forms a column.

2. Each observation forms a row.

3. Each type of observational unit forms a table. (p. 4) 
These basic principles go a long way toward ensuring that the clean data you share will be readily usable by other researchers. Characteristic 1 above is relatively straightforward, but note that this means you should not have 'combined' variables in your data. For example, in a psycholinguistic experiment where it is common to manipulate the grammaticality of stimulus sentences, it can be tempting to create a categorical variable called 'stim' with labels like 's01_u' and 's01_g' for "sentence one, ungrammatical" and "sentence one, grammatical, respectively. Doing so, however, is not tidy! The information should be broken up into two variables, each with its own column. For example, the 'stim' column should only have labels like 's01' indicating the sentence, and another column 'gram' for grammaticality should be created that features values such as ' $\mathrm{g}$ ' and ' $\mathrm{u}$ ' (or 1 and 0 , if you prefer).

Characteristic 2 complicates matters somewhat - what should be considered an observation? In a one-shot observational study, such as a survey, an observation is essentially the responses from a single person, so each person gets their own row. In a pre-post (quasi-)experimental design, however, each person is observed two times, so each person should get two rows. This type of data organization is commonly referred to as 'long' data, in contrast to 'wide' format data that adds more columns instead. That said, some flexibility here is reasonable; with a relatively simple dataset that has few variables, it is not a major problem to use a wide format and simply add another column for posttest scores. Some software used for analyzing test data, where one can think of each item response or rater-score combination as an observation, actually prefers wide-format data. But for many statistical programs, it will generally be preferred (if not required) that such within-person designs have multiple rows for each person, commensurate with the number of observations of variables of interest. This becomes even more crucial when analyzing longitudinal or time series data, where individual participants are 
observed several or several dozen times. My recommendation is that you share clean data that is in a format most conducive to running the primary analyses of your study. Don't make readers execute a series of data reshaping steps just to more closely examine your regression model diagnostic plots.

Characteristic 3 implies that different types of observations should get their own spreadsheets. For example, if you have a background survey and some textual indices of lexical sophistication in a study, you would have one spreadsheet with learner background data and one spreadsheet with lexical sophistication variables. These two spreadsheets, however, would need to be carefully linked with a common identifier for participants, and a preliminary step in the primary analyses of the study would include joining the two data sets. This recommendation of Wickham's (2014) is well-founded, especially when dealing with very large, complicated ensembles of data ('big data'), but in the context of most applied linguistics research, I would say that this recommendation can often be ignored when your study is relatively simple and/or the number of variables is more manageable.

\section{Variable Names}

Aside from the general shape of the data, it should be labelled in convenient, transparent ways. You'll want to use naming conventions that are easy to read for humans as well as computers.

For computers:

- Do not begin your variable names with numbers or other non-alphabetic characters. This will make many computer programs unhappy, and might force those programs to add some kind of special character anyway (e.g., "2 reading main idea" becomes ("x2 reading main idea" or ".2 reading main idea"). 
- When using numbers as part of variable names, including leading zeroes (e.g., "02” instead of just " 2 "). The number of leading zeroes depends on the largest number in your variable naming scheme, and ultimately the inclusion of leading zeroes will ensure that most computer programs will sort variables in the order you'd expect (e.g., 03, 13 instead of 13,3$)$.

- Avoid using empty spaces (created with the space bar) in variable names. Instead, use characters like dash (-) or underscore (_) (e.g., "02_reading_main_idea" instead of "02 reading main idea"). This ensures that your variable name will be easily readable by a wide range of computer programs.

- Aside from inserting spaces, avoid using special characters (e.g., ?, !, /) in variable names. Depending on the computer program, some of these special characters can get processed in unexpected ways.

For humans:

- Names should generally include some description of what the variable is, not just where it came from. "question_02" only tells you where the data came from, but “reading_question_02" lets you and any other potential users of your data know that it is a variable related to reading.

- Use descriptive words and/or intuitive abbreviations to avoid excessively long variable names. For example, "read" is a suitable abbreviation of "reading", and "q" for "question" is likely intuitive for most people, e.g. "read_q_02".

\section{Codebook/Data Dictionary}

Along with sharing your raw and clean data, it is extremely helpful for other researchers if you also share a guide to your data. A codebook or data dictionary is a document that provides 
details on what each variable in a (clean) dataset corresponds to. Generally, data dictionaries take the form of a table or spreadsheet. The first column is the name of each variable in the dataset. The second column can include a 'prettier' version of the variable name that could be used in published prose, tables, or figures, e.g., "Reading Comprehension Test - Question 2". Additional columns might include additional human-oriented, concise labels like "RQ2" that would work nicely in a table or a plot. A data dictionary may also include a more detailed description of what the variable represents. For example, a variable labeled 'speech_rate' might require some elaboration about (a) what the measure is based on (e.g., morae in L2 Japanese) and (b) how it was normed (e.g., per second). Last, but not least, it is extremely helpful when data dictionaries include information about the range of values a variable can take. For continuous numeric variables, this will often just be a range of values (e.g., "0-120”). For other variables, such as ordinal or categorical variables, numeric codes should be elaborated on. In the case of dichotomous reading test items, this would include " $0=$ incorrect, $1=$ correct". This information is especially critical in survey data, making it clear how verbal response options are numerically coded and which items are reverse coded.

Loewen et al.'s (2020) study on the statistical literacy of applied linguists features open data with an accompanying data dictionary (OSF link https://osf.io/ysj7p/). Figure 1 is a snapshot of their dictionary. The leftmost column contains variable names. Note that there are no spaces in variable names, and instead characters such as underscore are used. The Description column provides elaboration on what each variable represents and the Type column covers the data type associated with each variable, e.g., "string" for text data and "categorical" for nominal data (this information may not always be necessary, but it can remove any potential confusion for other researchers and/or help troubleshooting if a statistical program makes a wrong guess about the 
data type). Finally, the Codes column provides information on the possible labels (for categorical data) and scales for ordinal data. While Loewen et al. could have gone further in describing their dataset, for example by including some basic descriptive statistics associated with each variable (e.g., ranges for integer variables), this dictionary is sufficiently informative and is an attainable model for other researchers.

\begin{tabular}{|c|c|c|c|}
\hline \multicolumn{4}{|l|}{ Show rows with cells including: } \\
\hline Variable Name & Description & Type & Codes \\
\hline DegYear & year that highest degree was attained & integer & \\
\hline AcadPos & current academic position & categorical & $1=$ MA student, 2 = PhD student, 3 = Assistant Profes... \\
\hline AcadPos_text & alternative description for current academic position & string & \\
\hline Field & major field of study & categorical & $1=$ Applied Linguistics, $2=$ TESOL $/ \mathrm{TEFL}, 3=\mathrm{SLA}, 4 \ldots$ \\
\hline Field_Other_text & alternative description of major field of study & string & \\
\hline Inst & current institution type & categorical & 1 = University, 2 = Language school, 3 = Community ... \\
\hline Inst_text & alternative description of current institution type & string & \\
\hline Location & location of current institution & categorical & $1=\mathrm{N}$. America, 2 = Europe, $3=$ Australasia, $4=$ Asia, $\ldots$ \\
\hline Location_text & alternative description of current instifution location & string & \\
\hline Rsrchldent & degree of self-identification as a researcher & ordinal & $1=$ not at all, $6=$ Exclusively \\
\hline
\end{tabular}

Figure 1. Snapshot from Loewen et al.'s (2020) data dictionary, hosted on OSF

(https://osf.io/s5ryn/).

\section{Preparing Analysis Files for Others}

\section{What should you share?}

At the very minimum, you should share scripts for the primary analyses reported in your article, including scripts used to generate any results in appendices or supplements. These scripts will often be based on cleaned data. To further increase the credibility and replicability of your research, you should strongly consider sharing scripts used for processing and cleaning your data, especially if you are able to share your raw data. This creates a paper trail from the raw data to the data you used in analyses ultimately reported in the article. It also can help explain or avoid situations in which other researchers, working off of the same raw data, end up with different results due to not knowing the data cleaning procedures of the original study (e.g., Nicklin \& Plonsky, 2020). Finally, while not quite as necessary as primary analysis and data 
cleaning scripts, it is helpful to share scripts used to create data-based plots that are part of your article. While presumably not as common as statistical errors, it is possible to make plotting errors, such as setting plot limits too narrowly and excluding some data points from being displayed - hopefully you will catch such mistakes before you upload your script, but if not a careful and considerate reviewer might be able to alert you to the issue.

\section{Format Considerations}

You should share your scripts in formats that are useful and accessible. 'Useful' is straightforward - the script should be something that can be read into the analysis program and used to reproduce your findings. So if you ran an analysis in SAS, you should share an SAS script. 'Accessible' requires a little more thought. Will all of your readers and fellow researchers have access to the program that will allow them to view/use your script? In the case of proprietary, commercial software, probably not. But if such readers can still view your code, they can at least scrutinize your analyses and potentially replicate them using different software. So make sure that your shared scripts can be viewed/opened by others even if they are used with commercial software. Most analysis scripts are really just text files and can actually be opened with most common text editors (e.g., Notepad for Windows) - just make a note somewhere for your readers on how they can view the code. Alternatively, you can paste your code into a separate text file or PDF for easy viewing.

\section{Writing Good, Human-Friendly Code}

Just like the use of human- and computer-friendly variable names in your data is important, names in analysis scripts are also important to make your code human-readable and easier to follow. Providing notes and instructions in your code is another valuable practice to 
help ensure that others understand what each line of code does (and yourself, if you come back to code a year or two later).

\section{Names}

At some point, we've all been guilty of loading two datasets into a statistics program and referring to them as "d" and "d2". We have all probably run multiple statistical models and labelled them as "m1", "m2", "m3"... "m11". This is not best practice, especially for a script that you want to make accessible to others. Although more weight should be given to conciseness for names in analysis scripts compared to variable and file names, as you often need to refer to a dataset or model many, many times in a script, it is not too unreasonable to include some description: "read_mod" and "listen_mod" or "linear.mod" and "quad.mod" are preferable to "m1" and "m2".

\section{Annotation}

Above and beyond naming objects in your scrips, annotation via comments is the best way to ensure that other people will be able to make sense of your analysis in detail. And to be honest, it's the best way to ensure that you will be able to understand what you did when you come back to the script after a reviewer requests additional details or analyses. It will also come in handy much later - say, a year after publication when you need to prepare a tutorial or want to start working on a follow-up study. Generally, the easiest way to annotate your scripts is to include comments in the script itself. There are other options, though: You can create separate documents that walk readers through your code bit-by-bit. Sometimes these documents are included as article supplements and can be created simply enough in word processing programs (or for the especially tech-savvy, you can create such documents via markdown using $\mathrm{R}$ and RStudio; see example of R markdown in Liu \& De Cat, this volume). 
Many, if not all, statistical software packages allow you to write comments in scripts.

Comments are notes that are not read by the computer as a command - they exist solely for human readers. Comments are usually created by using a special character. In $\mathrm{R}$ you use \# and everything on the line that follows is a comment (other programs use other characters - in Winsteps, a program for Rasch measurement, a semicolon is used for commenting).

Consider the following example of R code (modified from Hui, 2020). If you have never used R before, you probably have no idea what this chunk of code does. If you are an experienced R user, you can probably follow along without too much trouble and understand the gist of what this chunk of code accomplishes. Even so, an experienced R user is unlikely to know exactly why this chunk of code is needed at first glance.

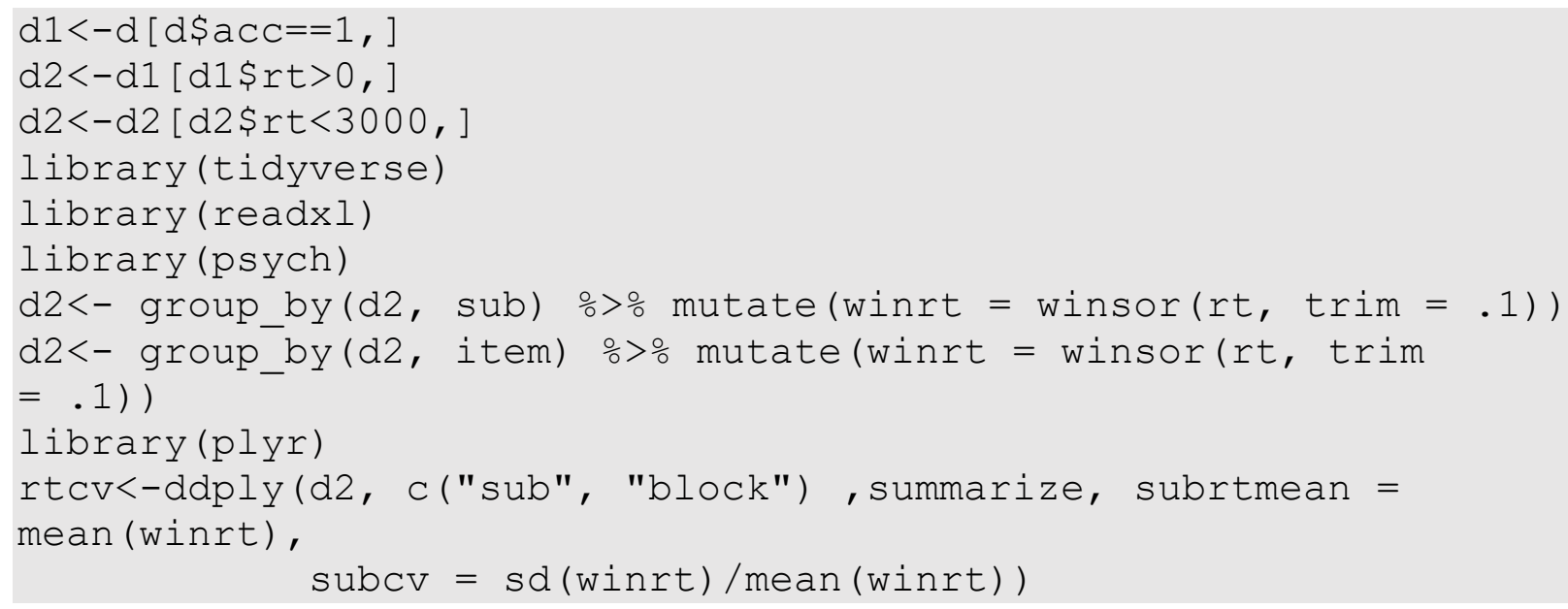

Now take a look at the original, well-commented and formatted version of the code in Hui's (2020) open analysis script:

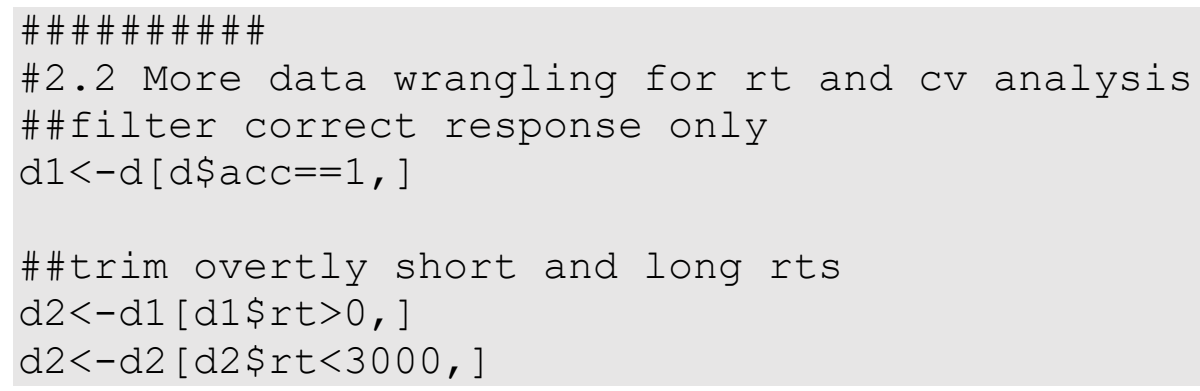




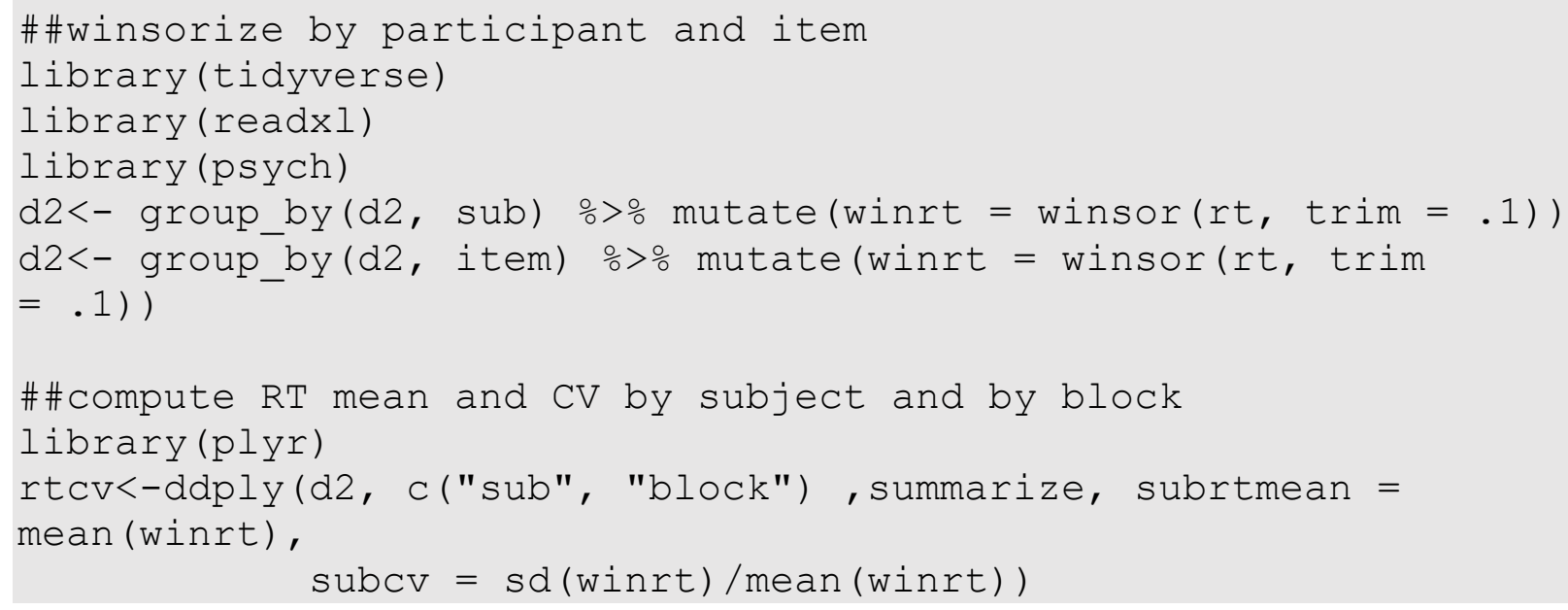

With these annotations, Hui's script is rather easy to follow, and we are provided with reasons for why we need each line of code. We now know that the code $d 1<-d[d \$ a c c==1$, ] is filtering out incorrect responses from a dataset. We can now understand that the code $\mathrm{d} 2<-$ group_by (d2, sub) $\frac{\circ}{0}$ mutate (winrt $=$ winsor $(r t, \operatorname{trim}=.1)$ ) is

Winsorizing (trimming) extreme reaction times for each participant. Hui’s liberal use of \# symbols and full-returns to visually break up the script is welcome. Note that he has also implemented some section numbering (“2.2") to organize his script - this is a very useful practice when your script is long (Hui's is over 500 lines) and paired with documentation of analysis steps (see the file 'ReadMe_Codebook.docx' hosted on Hui's OSF project site, https://osf.io/2ta7m/).

\section{Integrating Sharing into your Workflow}

\section{Before the study: Pre-registration}

If you are preregistering your study, that means you should have a very clear idea about what kind of data you will be collecting and should be able to define most of your key study variables in advance (see Huensch, this volume). And if you can define your variables, you can 
also give them sensible names and start building a data dictionary. A meticulous preregistration might even include a simulation or pilot study, replete with data and/or analysis scripts, so there is a great deal of potential to take early steps that will facilitate data and analysis sharing for the completed project.

\section{Get Organized}

Good organization can facilitate good data and analysis sharing practices: If you can't easily keep track of your data and analyses, you will probably be less likely and/or less able to share them in a useful way. Who among us has never made a copy of a file, done some work and saved it to the desktop, and then wondered where that work went when logging on to a different computer? For this reason, I recommend using some sort of cloud storage that allows you to keep your files in one place regardless of what machine you are using - your office computer, a laptop, a lab computer, etc. There are a number of options here, such as Google Drive and Dropbox, so choose one that works for your budget and data security concerns.

Getting organized isn't only a matter of using a cloud storage service. Within that cloud storage, a sensible folder structure will help prevent files from getting misplaced and facilitate the eventual sharing process. While there are many sensible ways to organize computer files for a research project (e.g., Chapter 2 in Gries, 2013; Chapter 2 in Winter, 2020), the following example can be a useful model, especially if you haven't found a structure you like yet:

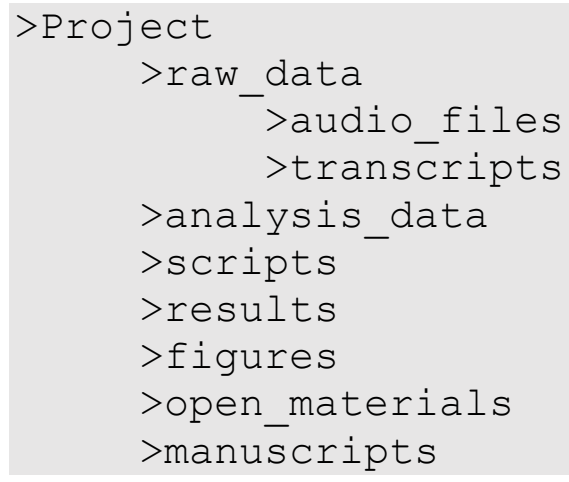


In this structure, the top level 'Project' could be named something more descriptive, but the point is that it's the top-level file in the structure that houses all other files. The 'raw_data' folder houses your raw data files. In this example, there are subfolders for audio files and transcripts; naturally other projects will differ. Importantly, the data in the 'raw_data' folder and subfolders should generally not be edited, overwritten, or otherwise altered. 'analysis_data', on the other hand, is where cleaned and aggregated data should be located.

The 'scripts' folder is where all data cleaning and analysis scripts are stored. If you are undertaking an especially complicated project, you might want to use subfolders within the scripts folder, but in many cases that will not be necessary. Finally, the 'results' and 'figures' folders are where you can store the output of your analyses, with 'figures' omitted if you aren't planning to generate any.

The penultimate folder in the structure, 'open_materials', is optional. If all of your data are anonymized and you want to share everything, this folder will likely be unnecessary. Otherwise, this folder will house files you are able to share. If you are not sharing any raw data, you might just have a few files from your 'analysis_data' and 'scripts' folder that you drop in here. If you are able to share anonymized (i.e., not strictly original) raw data and decide it would be appropriate to share a large number of scripts, you might place copies of your raw_data, analysis_data, and scripts folders into the open_materials folder and then anonymize all data and scripts, including the removal of any information that could identify participants or authors (so that you can share with journal reviewers - more on this later). Finally, the 'manuscripts' folder is there to organize the write-up of the project, and you might use subfolders like 'initial submission' and 'revision_1' to keep things organized as you move through the publication process. 


\section{Use Open Science Framework or another Project Management Solution}

Project management platforms can serve as both a storage back-up and a means for sharing your data and analysis scripts. The Open Science Framework (OSF) is the project management system I've chosen to highlight for this chapter, as it is a user-friendly and highly functional option. It's incredibly easy to upload data and scripts for personal backup and sharing with others, plus you can also upload instruments, supplements, and other relevant materials (Soderberg, 2018). OSF allows a sizable amount of data and other materials to be stored for free (5 GB for private, non-shared projects and 50 GB for publicly-shared projects). Generally speaking, for applied linguists only very large corpora and/or large amounts of audio/video data will stretch those limits. This means that you should be able to use OSF as a back-up for your project folder that you created in your cloud storage (and you can even integrate several cloud storage services with OSF, but that is more of an advanced topic).

For very large or particularly sensitive datasets (which you might want to partially restrict access to), more robust data repository services like ICPSR

\section{(https://www.icpsr.umich.edu/web/pages/) and Dataverse (https://dataverse.org/) are available,} but might incur some cost or require institutional membership depending on which services you wish to use. Finally, while you can share data through public Google Drive or Dropbox links, I generally recommend against doing so, as the hosting of files is contingent on an account being active. Inactive Dropbox accounts can be deleted after some time, and if your institution decides to switch from Google to Microsoft for email and cloud services, for example, your public Drive links could be jeopardized. 


\section{Final Data and Analysis Check}

Before sharing your data and analyses, you will want to run through everything one last time and make sure the results line up with the manuscript you are planning to submit. The best way to do this is to download the materials that you've uploaded to OSF (or another site), place them in your desktop or documents folder, and then do a 'fresh' run-through of your analyses using the data and scripts you plan to share.

Although you can do this on your own, it's even better if you can get someone else to help check and make sure everything works. The benefit of having someone else run through your data and analyses is that you can troubleshoot any technical problems and get a fresh set of eyes on the data and scripts to screen for identifying information or comments in the code that are unclear. A common technical issue is file location - for instance, you might have a file path in a script that only works on your computer. If you're using R, it's easy to forget to include code for loading necessary packages in your script, especially if you are typically working with multiple scripts at once or generally prefer to load packages using through menus. If you have collaborators, make this a team effort - the person who worked mostly on the literature review and instrument development, for example, could be asked to try out the shared data and analysis files to make sure results line up with what is reported in the manuscript. You can also ask trusted colleagues to try things out before you make a sharing link available to reviewers or the public. In my experience, this step doesn't take a lot of time and leads, quite often, to the identification of one or more errors or infelicities. 


\section{Insert Your (Anonymous) OSF Link in the Manuscript}

The final step is to include a link to your data and scripts in your paper. Most journals should allow you to include such a link somewhere in the manuscript. I personally prefer having the link in the 'Current Study' section or at the beginning of the 'Methods' section.

On OSF, you can share a link associated with your entire project, which in many cases is the simplest and best way to go. However, if you've been using some of OSF's features to support collaboration, like wiki pages for drafting parts of an article or file storage for meeting notes, you might not want to share the entire project. In that case, you can create project components that allow for sharing only the specific parts of the project. For example, you could designate your 'raw_data', 'analysis_data', and 'scripts' folders as part of a component meant for sharing.

One concern about sharing data and scripts is that your sharing link and data hosting solution might compromise anonymity. With few journals in applied linguistics using singleblind (e.g., Bilingualism: Language and Cognition) or completely open review processes (e.g., special issues in the Frontiers family of journals), this is a common hurdle. Luckily, OSF makes it possible to share your data and analysis scripts anonymously. You'll still need to ensure that the files you are sharing do not contain information that identifies any authors, but the link itself and the OSF project page it directs to will not list author/contributor information (Figure 2, left). When you submit your manuscript for review, you can include the anonymous link, and once it has been accepted, you can insert the standard link in the manuscript (Figure 2, right). The standard link is important because it will allow people to search for your data and you can provide helpful information like the reference and DOI of the published article. After inserting your sharing link in the final version of the article, the process is complete! Well, almost - after 
publication, it's also strongly recommended to submit your study's instruments and other materials, including data/analysis script files or links to them, to the IRIS database (https://www.iris-database.org), which provides much better indexing and search tools for applied linguists compared to OSF.

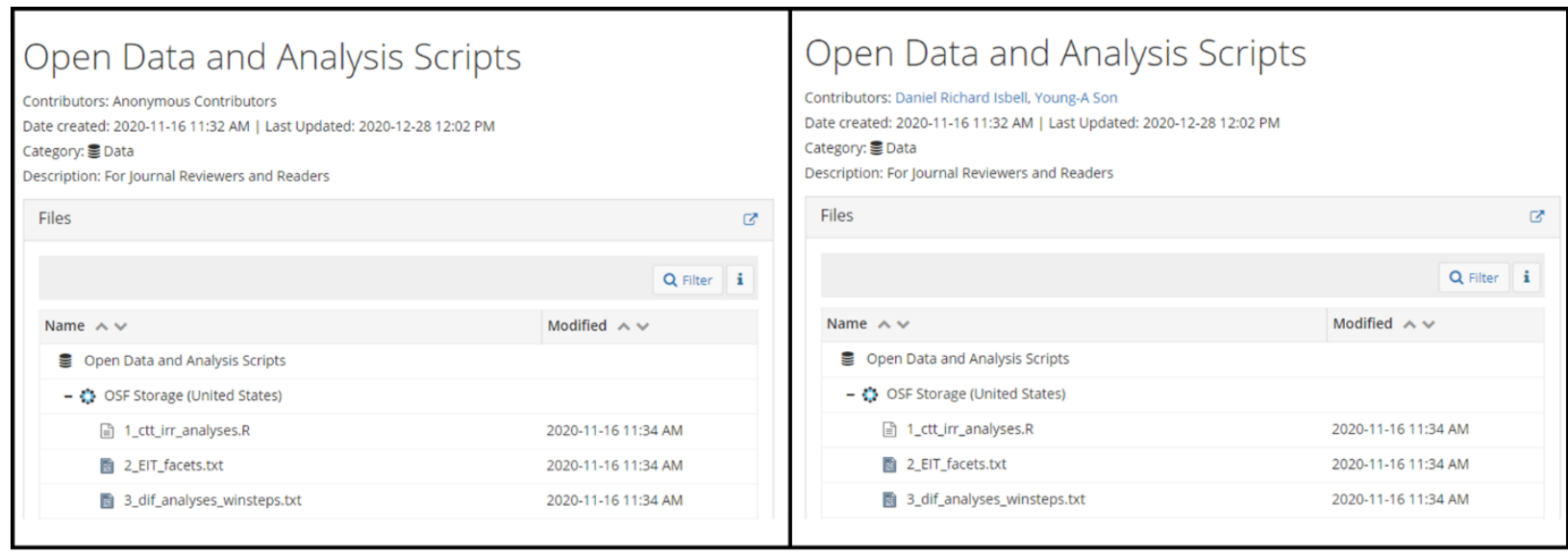

Figure 2. Comparison of anonymous (left) and standard (right) views of the OSF project associated with Isbell and Son (2021).

\section{Conclusion}

This chapter has made the case for and provided guidance on sharing both data and analysis scripts used in applied linguistics research. I have tried to present an approach to sharing that is minimally burdensome, but I nonetheless worry that some readers will see sharing as extra work that is technically unnecessary for conducting and publishing research. Such readers would not be incorrect: As it currently stands, none of this is strictly necessary. My own publication record is an attestation, with numerous gaps in data and analysis sharing. In fact, I do not yet have a publication that faithfully follows every single recommendation that I have made in this chapter (a recent publication comes close, but it is missing a data dictionary). But falling short of the bar in the past should not preclude any of us from doing things more transparently and in line with Open Science principles now and going forward, or even looking back - there's nothing to 
stop you from sharing data and analysis scripts from your published studies. First, there are some immediate benefits: Sharing your data and analyses will (a) improve the credibility of research that you've invested time, money, and effort in and (b) allow you to earn some nifty Open Science badges, which provide a recognizable mark of rigor that evaluators (e.g., funding agencies, awards committees, tenure and promotion committees) might take notice of. Second, starting to share now will future-proof your research practices. As the field continues to evolve, I predict you will find that more and more journals, funding agencies (Brysbaert, this volume), and graduate training programs (Hui and Huntley, this volume) will make sharing generally required. My hope is that 10 years from now, graduate students and early career researchers will read this chapter with some wonderment at the idea that basic sharing of data and analysis scripts in quantitative applied linguistics research was ever optional. 


\section{References}

Ahn, S., Chang, C. B., DeKeyser, R., \& Lee-Ellis, S. (2017). Age effects in first language acquisition: Speech perception by Korean-English bilinguals. Language Learning, 67(3), 694-733. https://doi.org/10.1111/lang.12252

Al-Hoorie, A. H., \& Vitta, J. P. (2019). The seven sins of L2 research: A review of 30 journals' statistical quality and their CiteScore, SJR, SNIP, JCR impact factors. Language Teaching Research, 23(6), 727-744. https://doi.org/10.1177/1362168818767191

American Statistical Association. (1999). Ethical guidelines for statistical practice. Author. https://www.amstat.org/asa/files/pdfs/EthicalGuidelines.pdf

Broman, K. W., \& Woo, K. H. (2018). Data organization in spreadsheets. The American Statistician, 71(1), 2-10. https://doi.org/10.1080/00031305.2017.1375989

Buchanan, E. M., Crain, S. E., Cunningham, A. L., Johnson, H. R., Stash, H., Papadatou-Pastou, M., Isager, P. M., Carlsson, R., \& Aczel, B. (2021). Getting started creating data dictionaries: How to create a shareable data set. Advances in Methods and Practices in Psychological Science, 4(1), 1-10. https://doi.org/10.1177/25152459209

Gries, S. Th. (2013). Statistics for linguistics with R: A practical introduction. De Gruyter Mouton.

Hui, B. (2020). Processing variability in intentional and incidental word learning: An extension of Solovyeva and DeKeyser (2018). Studies in Second Language Acquisition, 42, $327-$ 357. https://doi.org/10.1017/S0272263119000603

Hussong, A. M., Curran, P. J., \& Bauer, D. J. (2013). Integrative data analysis in clinical psychology research. Annual Review of Clinical Psychology, 9, 61-89. https://doi.org/10.1146/annurev-clinpsy-050212-185522 
Isbell, D. R., \& Son, Y-A. (2021). Measurement properties of a standardized elicited imitation test: An integrative data analysis. Studies in Second Language Acquisition. Advance online publication. https://doi.org/10.1017/S0272263121000383

Loewen, S., Gönülal, T., Isbell, D. R., Ballard, L., Crowther, D., Lim, J., Maloney, J., \& Tigchelaar, M. (2020). How knowledgeable are applied linguistics and SLA researchers about basic statistics? Data from North America and Europe. Studies in Second Language Acquisition, 42(4), 871-890. https://doi.org/10.1017/S0272263119000548

Loewen, S., \& Hui, B. (2021). Small samples in instructed second language acquisition research. Modern Language Journal, 105(1), 187-193. https://doi.org/10.1111/modl.12700

Meyer, M. N. (2018). Practical tips for ethical data sharing. Advances in Methods and Practices in Psychological Science, 1(1), 131-144. https://doi.org/10.1177/251524591774765

Nicklin, C., \& Plonsky, L. (2020). Outliers in L2 research in applied linguistics: A synthesis and data re-analysis. Annual Review of Applied Linguistics, 40, 26-55. https://doi.org/10.1017/S0267190520000057

Saunders, B., Kitzinger, J., \& Kitzinger, C. (2015). Anonymising interview data: Challenges and compromise in practice. Qualitative Research, 15(5), 616-632. https://doi.org/10.1177/1468794114550439

Simmons, J. P., Nelson, L. D., \& Simonsohn, U. (2011). False-positive psychology: Undisclosed flexibility in data collection and analysis allows presenting anything as significant. Psychological Science, 22(11), 1359-1366. https://doi.org/10.1177/0956797611417632

Simonsohn, U. (2013). Just post it: The lesson from two cases of fabricated data detected by statistics alone. Psychological Science, 24(10), 1875-1888. https://doi.org/10.1177/0956797613480366 
Soderberg, C. K. (2018). Using OSF to share data: A step-by-step guide. Advances in Methods and Practices in Psychological Science, 1(1), 115-120.

Wickham, H. (2014). Tidy data. Journal of Statistical Software, 59(10), 1-23.

Winter, B. (2020). Statistics for linguists: An introduction using R. Routledge. 